\title{
Characterization of a Ralstonia solanacearum Operon Required for Polygalacturonate Degradation and Uptake of Galacturonic Acid
}

\author{
Enid T. González and Caitilyn Allen \\ Department of Plant Pathology, University of Wisconsin-Madison, 1630 Linden Drive, Madison 53706 U.S.A.
}

Submitted 24 September 2002. Accepted 23 January 2003.

\begin{abstract}
The bacterial wilt pathogen Ralstonia solanacearum produces three extracellular polygalacturonases (PGs): PehA, PehB, and PehC. All three PGs hydrolyze pectin's polygalacturonic acid backbone, but each releases different reaction products. PehA and PehB contribute significantly to pathogen virulence, probably by facilitating root invasion and colonization. To determine the collective contribution of PGs to virulence and saprophytic survival, we cloned, characterized, and mutated the $R$. solanacearum pehC gene, which encodes a distinctive monogalacturonate-releasing exo-PG. The virulence of a pehC mutant on tomato was indistinguishable from that of its wild-type parent; thus, this exo-PG alone does not contribute significantly to wilt pathogenesis. Unexpectedly, a completely PG-deficient triple $p e h A / B / C$ mutant was slightly more virulent than a pehA/B mutant. PehC may degrade galacturonide elicitors of host defense, thereby protecting the pathogen from plant antimicrobial responses. A galacturonate transporter gene, exuT, is immediately downstream of pehC and the two genes are co-transcribed. It has been hypothesized that galacturonic acid released by PGs from plant cell walls nourishes bacteria during pathogenesis. To separate the pectolytic and nutrient-generating roles of the PGs, we made an exuT mutant, which still produces all three isozymes of PG but cannot uptake PG degradation products. This exuT mutant had wild-type virulence on tomato, demonstrating that metabolism of galacturonic acid does not contribute significantly to bacterial success inside the plant.
\end{abstract}

Additional keywords: pectolysis, polygalacturonase.

Pectic polymers, the primary constituents of the higher plant middle lamella and primary cell wall, are chains of galacturonic acid, often branched and decorated with other sugars (Prade et al. 1999). Pectins pose a physical barrier to invading microbes, they offer an abundant carbon source inside the plant host and in the rhizosphere, and pectin degradation products can be potent elicitors of plant defense responses (Davis et al. 1984; Esquerre-Tugaye et al. 2000; Vidal et al. 1998). The fact that most cellular plant pathogens can degrade pectic substances suggests that these enzymes contribute to the fitness of plant-associated microbes. Nonetheless, determining the precise role of pectin-degrading enzymes in the life history of

Corresponding author. C. Allen; Telephone: (608) 262-9578; Fax: (608) 263-2626; E-mail: cza@ plantpath.wisc.edu

DNA sequence data of the pehC-exuT operon is available in the GenBank database under accession number AF417111. plant pathogens has not been straightforward, in part because these microbes often express multiple functionally redundant pectinase isozymes. The best-studied set of pectinases belong to the soft-rot pathogen Erwinia chrysanthemi, which produces dozens of assorted pectinases with subtle differences in mode of cleavage, substrate preference, regulation, and conditions for optimal activity (Hugouvieux-Cotte-Pattat et al. 1996). Even non-soft-rotting pathogens, like the fungus Cochliobolus carbonum, carry several families of apparently redundant pectinase genes (Scott-Craig et al. 1990, 1998). Because it makes only three functionally distinct polygalacturonases (PGs), the bacterial wilt pathogen Ralstonia (formerly Pseudomonas) solanacearum offers a good model system for exploring the role of pectolysis in plant-associated bacteria.

$R$. solanacearum, a soilborne, gram-negative plant pathogen, attacks an unusually wide range of plant hosts. This pathogen is responsible for extensive annual losses of subsistence and cash crops throughout the tropical and warm temperate world (Hayward 1991). R. solanacearum invades its host plants through the roots via wounds or secondary root emergence points, infects the root cortex, and then enters host xylem vessels (Vasse et al. 1995). Once in the xylem, the bacteria multiply and progress quickly upward through the plant vascular system, causing wilting and eventual plant death.

$R$. solanacearum virulence factors are quantitative, each one adding a degree of pathogen aggressiveness. Many of the known virulence factors are secreted, including an extracellular polysaccharide (EPS I), an endoglucanase, and three PGs (Denny and Baek 1991; Huang and Allen 1997; Roberts et al. 1988; Schell et al. 1988). Bacterial motility, both twitching motility mediated by type-IV pili and flagellar swimming motility, also contributes to virulence (Liu et al. 2001; Tans-Kersten et al. 2001). Expression of these factors is controlled by a complex regulatory cascade that responds to bacterial cell density and possibly to other signals as well (Schell 2000). In addition, a type III secretion system translocates effector proteins of unknown function into host cells in response to pathogen-plant cell contact; mutation of the type III secretion system drastically reduces virulence (Aldon et al. 2000; VanGijsegem et al. 1995). The genomic sequence of $R$. solanacearum GMI1000 contains nearly 200 known or potential virulence factor genes, reinforcing the multifactorial nature of virulence in this pathogen (Salanoubat et al. 2002).

A consortium of three plant cell wall-degrading PGs apparently facilitates plant invasion by $R$. solanacearum. Although all three PGs hydrolytically cleave the pectic polymer, they have distinct reaction products. PehA (also known as PglA), an endo-PG (E.C. 3.2.1.82), cleaves the pectic polymer internally at random, releasing large oligomers, mainly trimers of galac- 
turonic acid (galUA) (Allen et al. 1991; Roberts et al. 1988). PehB, an exo-poly- $\alpha$-D-galacturonosidase (E.C. 3.2.1.82), and PehC, an exo-PG (E.C. 3.2.1.67), cleave the pectic polymer at the nonreducing end of pectin, releasing dimers and monomers of galUA, respectively (Huang and Allen 1997). Site-directed mutants lacking PehA and PehB are severely reduced in virulence on eggplant and unwounded tomato, and PehA/PehB-deficient mutants cannot colonize host stems as well as the wildtype parent strain, presumably because PG-mediated degradation of pectic gels, middle lamellae, cell walls, and pit membranes helps bacteria penetrate plant roots and colonize vessels (Huang and Allen 1997, 2000). To date, the lack of a completely PG-deficient strain $\left(p e h A^{-} p e h B^{-} p e h C^{-}\right)$has made it impossible to determine the collective contribution of PGs to bacterial wilt virulence or saprophytic fitness. Therefore, we decided to isolate and mutate the gene encoding the last of the three extracellular PGs secreted by $R$. solanacearum, PehC.

Little is known about the structure or biological function of exo-PGs from bacteria. Aspergillus tubingensis, Fusarium oxysporum f. sp. lycopersici, and other fungi produce well-characterized exo-PGs, though their biological role remains uncertain (Kester et al. 1996; Lang and Dornenburg 2000; Pietro and Roncero 1996). A single bacterial exo-PG (from a Bacillus sp.) has been described, but its DNA and protein sequence is not known, nor has its biological role been determined (Kobayashi et al. 2001).

The transition from soil resident to xylem-inhabiting plant pathogen requires $R$. solanacearum to adapt to a strikingly different nutritional environment. Plant xylem fluid is not considered a nutritionally rich habitat (Bialczyk and Lechowski 1995; Senden et al. 1992). In addition to macerating plant cell walls and pectic gels, PGs can help bacteria harvest nutrients from plant cell walls. Degradation of pectic polymers releases large amounts of galUA, as well as lesser amounts of other sugars, such as galactose and arabinose (Prade et al. 1999). R. solanacearum K60 possesses a complete metabolic pathway to catabolize pectin breakdown products, because it can grow on both polygalacturonate (PGA) and galUA as sole carbon sources (Tans-Kersten et al. 1998). Therefore, we hypothesized that
PGs provide $R$. solanacearum with a significant nutrient source in the form of galUA during growth inside the plant host.

To test this hypothesis, we created a mutant that is fully pectolytic but cannot transport galUA, thereby separating the macerating function of $R$. solanacearum PGs from any role they might play in generating a carbon source. This mutant allowed us to determine the contribution of PG-generated nutrients to the ability of $R$. solanacearum to wilt and kill plants.

\section{RESULTS}

Isolation of the $R$. solanacearum pehC gene.

We used nucleotide sequence information from the genome of closely related $R$. solanacearum GMI1000 to design primers that amplified an internal portion of a PG gene similar to those from plants, fungi, and bacteria, but distinct from the two previously characterized $R$. solanacearum PGs, PehA, and PehB. The resulting 300-bp DNA fragment hybridized to two cosmids in a strain K60 genomic library; one, designated p1-46, was chosen for further analysis. A 4.8-kb BamHI fragment from cosmid p1-46 was subcloned to create pPehC1, which was sequenced (Table 1). This clone contained two open reading frames (ORFs) separated by $75 \mathrm{bp}$. The first ORF was predicted to encode a protein $75 \%$ identical to a putative PG in the $R$. solanacearum GMI1000 sequence (GenBank accession number NC003296). Named pehC, this ORF is 2,040 bp long, and encodes a predicted protein of 679 amino acids, molecular weight of $70.355 \mathrm{kDa}$, and a pI of 6.31. This is in good general agreement with the previously observed physicochemical characteristics of this enzyme (Huang and Allen 1997). The predicted protein sequence of PehC contains a PG active site from amino acid 445 to 459 , which classes the enzyme as a family 28 glycosyl hydrolase. Additional protein sequence analysis showed that PehC harbors four strictly conserved motifs (amino acids 398_NTD, 418_DD, 452_GHG, and 490_RIK) and apparently has the conserved tyrosine residue (511_Y) characteristic of family 28 glycosyl hydrolases (Markovic and Janecek 2001). CLUSTAL protein sequence alignment analysis of the predicted PehC protein suggests that this exo-PG differs

Table 1. Strains table

\begin{tabular}{|c|c|c|}
\hline Designation & Relevant characteristics $^{\mathbf{a}}$ & Reference \\
\hline \multicolumn{3}{|l|}{ Escherichia coli } \\
\hline $\mathrm{DH} 5 \alpha$ & F endA1 relA $\phi 80$ lacZ $\Delta$ supE44 thi-1 recA1 gyrA96 & Hanahan 1983 \\
\hline \multicolumn{3}{|l|}{ Erwinia chrysanthemi } \\
\hline SF1602 & exuT::Km ${ }^{\mathrm{r}}$ & Haseloff et al. 1998 \\
\hline \multicolumn{3}{|l|}{ Ralstonia solanacearum } \\
\hline K60 & Wild-type race1, biotype 1 & Kelman 1954 \\
\hline K71 & pehR::Tn3-uidA $\mathrm{Km}^{\mathrm{r}}$ & Allen et al. 1997 \\
\hline K60-P409 & pehC $:$ pPehC6 $\mathrm{Km}^{\mathrm{r}} \mathrm{Amp}^{\mathrm{r}}$ & This study \\
\hline K60-309 & pehA $: \Omega$ pehB $:$ aacC1 $\mathrm{Sm}^{\mathrm{r}} \mathrm{Gm}^{\mathrm{r}}$ & Huang and Allen 1997 \\
\hline K60-409 & pehC::aphA-3 $\mathrm{Km}^{\mathrm{r}}$ & This study \\
\hline K60-T09 & exuT::aacC1 $\mathrm{Gm}^{\mathrm{r}}$ & This study \\
\hline K60-709 & 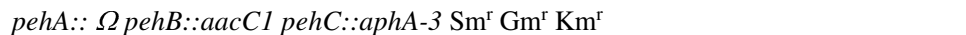 & This study \\
\hline \multicolumn{3}{|c|}{ 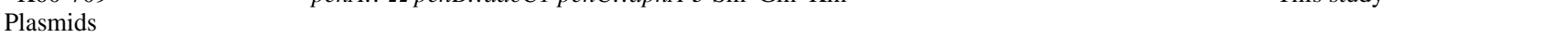 } \\
\hline pBluescript II SK (+) & $\mathrm{Ap}^{\mathrm{r}}$ & Stratagene, Inc., LaJolla CA \\
\hline pLAFR3 & $\mathrm{Tc}^{\mathrm{r}}$ & Staskawicz et al. 1986 \\
\hline pUC18K & $\mathrm{Km}^{\mathrm{r}}$ & Menard et al. 1993 \\
\hline pUCGM & $\mathrm{Gm}^{\mathrm{r}}$ & Schweizer 1993 \\
\hline p1-46 & 20-kb K60 genomic DNA fragment containing pehC-ехuT in pLAFR3. $\mathrm{Tc}^{\mathrm{r}}$ & This study \\
\hline pCR1 & 1.7-kb ApaI fragment containing exuT from p1-46 in pBSSK $(+)$. Ap $^{\mathrm{r}}$ & This study \\
\hline pExuT3 & $\begin{array}{l}\text { 1.7-kb ApaI fragment from pCR } 1 \text { with } 0.8-\mathrm{kb} \mathrm{Gm}^{\mathrm{r}} \text { cassette insertion from pUCGM } \\
\text { in pBSSK }(+) \Delta B a m \mathrm{HI} \mathrm{Ap}^{\mathrm{r}} \mathrm{Gm}^{\mathrm{r}}\end{array}$ & This study \\
\hline pPehC1 & 4.8-kb BamHI fragment containing pehC from p1-46 in pBSSK(+). Ap ${ }^{\mathrm{r}}$ & This study \\
\hline pPehC9 & & \\
\hline & from pUC18K in pBSSK(+) $\Delta C l a I . \mathrm{Km}^{\mathrm{r}} \mathrm{Ap}^{\mathrm{r}}$ & This study \\
\hline $\begin{array}{l}\text { pPehC10 } \\
\text { pPehC11 }\end{array}$ & & This study \\
\hline pPehC11 & & \\
\hline
\end{tabular}

\footnotetext{
${ }^{a}$ Ap, ampicillin; Tc, tetracycline; Km, kanamycin; Gm, gentamycin; Sm, streptomycin.
} 
significantly from its fungal counterparts, with only approximately $11 \%$ identity to exo-PGs from A. tubigensis, Botryonia fuckeliana, and $F$. oxysporum (GenBank accession numbers X99795, AF145229, and AF083075, respectively). PehC also is quite distinct from the other $R$. solanacearum $\mathrm{PGs}$, with only $15.7 \%$ identity to endo-PG PehA and $13.4 \%$ identity to PehB (GenBank accession numbers M33692 and U6101, respectively). Initial screens for heterologous PehC expression in Escherichia coli, detected as pit formation on pectate semisolid media, were unsuccessful. Using a more sensitive thin-layer chromatography (TLC) reaction product analysis, we determined that cell-free extracts of Escherichia coli harboring pehC on pPehC1 can release mono-galacturonate from polygalacturonic acid (PGA) substrate, demonstrating biochemically that the cloned pehC gene encodes exo-PG activity (Fig. 1, lane 9). In addition, TLC analyses also revealed that these PehC-containing extracts can cleave trigalacturonic acid substrate to release di- and monogalacturonate, and can cleave digalacturonate to release monogalacturonate, suggesting that PehC can digest the small oligogalacturonides released by PehA and PehB, as well as the long-chain polymers of PGA (data not shown).

\section{pehC and exuT are co-transcribed in an operon.}

A second partial ORF directly downstream of pehC was predicted to encode a protein similar to ExuT, a galacturonic acid transporter from both Escherichia coli (56\% identity, GenBank accession number P42609) and from E. chrysanthemi (52\% identity, GenBank accession number AAB70881.1). To isolate the entire exuT gene, a 1.7-kb ApaI fragment containing the exuT ORF was subcloned from cosmid p1-46, creating plasmid pCR1. Sequence analysis revealed that exuT is $1,320 \mathrm{bp}$ long and appears to encode a $46.9-\mathrm{kDa}$ protein. BLASTX protein analysis suggested that ExuT is likely a sugar transporter containing 10 putative transmembrane helices. No obvious promoter is apparent in the 75-bp intergenic region separating exuT from pehC, although a putative ribosome binding site $\left(5^{\prime}-\right.$ AGGAGGC-3') is present $10 \mathrm{bp}$ upstream from the exuT start codon. A mutant carrying a polar insertion in pehC (K60-P409) has an exuT mutant phenotype; that is, it is unable to grow on minimal media containing galacturonic acid as a sole carbon source. This functional analysis offers genetic evidence that pehC and exuT form an operon (Fig. 2).
Construction and characterization

of pehC mutant strain K60-409 and $\mathrm{PG}^{-}$strain K60-709.

Mutations in pehC could be polar onto exuT; therefore, we created a nonpolar mutation in pehC using the aphA-3 nonpolar mutagenesis cassette (Menard et al. 1993). The pehC::aphA-3 construct was introduced into $R$. solanacearum K60 by allelic replacement mutagenesis to create strain K60-409. Southern blot analysis and DNA sequencing of insertion junctions verified the correct, in-frame insertion of the cassette in the $R$. solanacearum K60 genome. To confirm biologically that the pehC mutation was functionally nonpolar, we verified that this mutant can grow on galUA as a sole carbon source, an indication of intact ExuT function (Fig. 3A). Reaction product analysis of PG activity in K60-409 culture supernatants confirmed that this mutant had the predicted pehC profile, releasing no monogalacturonate but only di- and trigalacturonate, the reaction products of PehB and PehA, respectively (Fig. 1, lane 3). When the pehC-exuT operon was present in trans, it restored the ability of K60-409 to generate monogalacturonate from PGA (Fig. 1, lane 4). We introduced the pehC::aphA-3 construct into pehApehB mutant $\mathrm{K} 60-309$ to create a fully $\mathrm{PG}^{-} \mathrm{mu}-$

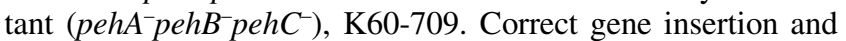
location was verified via Southern blot analysis. K60-709 had intact ExuT function, because it grew on minimal medium containing galUA as a sole carbon source (data not shown). K60709 generated no detectable PG reaction products in the sensitive TLC reaction product analysis, demonstrating that this mutant was completely nonpectolytic (Fig. 1, lane 6).

exuT encodes a galacturonate transporter.

To biologically test the hypothesis that the gene downstream of pehC encoded a galUA transporter, we mutated it by inserting a gentamycin resistance gene cassette into the unique BamHI site 797 bp 3' from the putative exuT start site. The resulting exuT::aacCl construct was introduced into the $R$. solanacearum K60 genome by allelic replacement, generating strain K60-T09. The insertion specificity was verified by Southern blot analysis (data not shown). Although this mutant grew as well as its wild-type parent in planta and in minimal media plus glucose as a sole carbon source (data not shown), it could not grow on galUA as a sole carbon source, indicating lack of exuT transport function (Fig. 3A). However, the wild-type pehC-exuT operon sup-

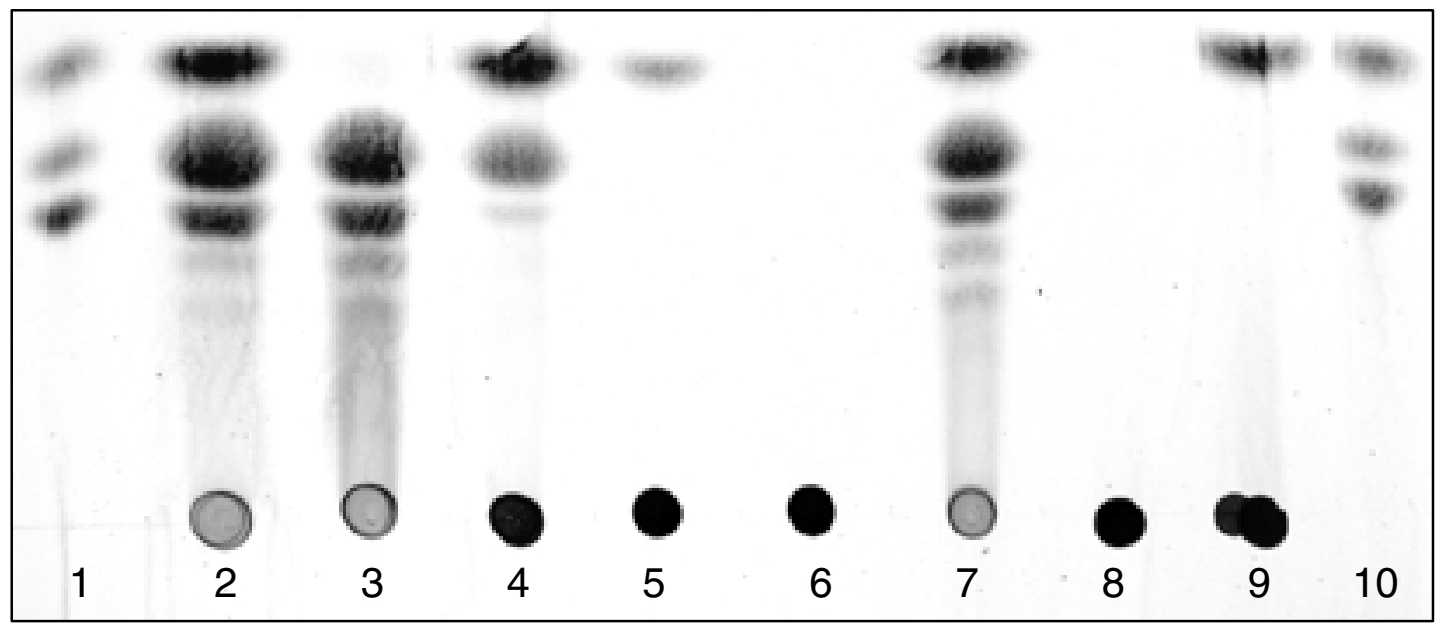

Fig. 1. Thin-layer chromatography analysis of polygalacturonase reaction products from pehC mutant K60-409 and exuT mutant K60-T09. Extracellular protein $(0.1 \mathrm{mg}$ ) from spent culture supernatants of Ralstonia solanacearum wild-type strain K60 (lane 2), pehC mutant K60-409 (lane 3), pehC mutant K60-409 plus pehC-exuT containing plasmid pPehC11 (lane 4), pehApehB mutant K60-309 (lane 5), pehApehBpehC mutant K60-709 (lane 6), and exuT mutant K60-T09 (lane 7) was incubated with $0.4 \%$ (wt/vol) polygalacturonic acid in $50 \mathrm{mM}$ morphonlinethanesulfonic acid, $\mathrm{pH} 5.7$, for $1 \mathrm{~h}$ at $45^{\circ} \mathrm{C}$. Similarly, cell-free extracts or spent supernatants of Escherichia coli harboring empty vector pBluescript or pPehC1 were treated as previously described (lanes 8 and 9, respectively). Pure mono-, di-, and trigalacturonic acid standards (4 $\mu \mathrm{g}$ each) were spotted on the plate for reference (lanes 1 and 10). 
plied in trans restored wild-type growth of K60-T09 on galUA (Fig. 3B). Importantly, K60-T09 still produced all three pectic enzymes, generating mono-, di-, and trigalacturonate acid from the PGA substrate in a polygalacturonase reaction product profile indistinguishable from that of the wild-type strain (Fig. 1, lane 7). Moreover, the R. solanacearum exuT gene could heterologously complement an $E$. chrysanthemi exuT mutant. Plasmid pPehC10, containing the entire pehC-exuT operon, restored the ability of E. chrysanthemi exuT mutant SF1602 to grow on galUA as a sole carbon source. This result offers additional evidence that exuT encodes a functional galacturonate transporter.
ExuT is the only transporter of polygalacturonate breakdown products.

To make certain that no other galacturonate oligomers were being transported into the bacterial cell, we assayed the ability of exuT mutant K60-T09 to grow on PGA as a sole carbon source. This mutant could not grow in modified Boucher's minimal media without yeast extract (BMM) + $0.2 \%$ (wt/vol) PGA, suggesting that, although the bacterium's extracellular PGs released mono-, di-, and trigalacturonate, the bacterium had no mechanism of transport for these three reaction products (Fig. 3B). Only when exuT was provided in trans could K60-T09 grow on this carbon source

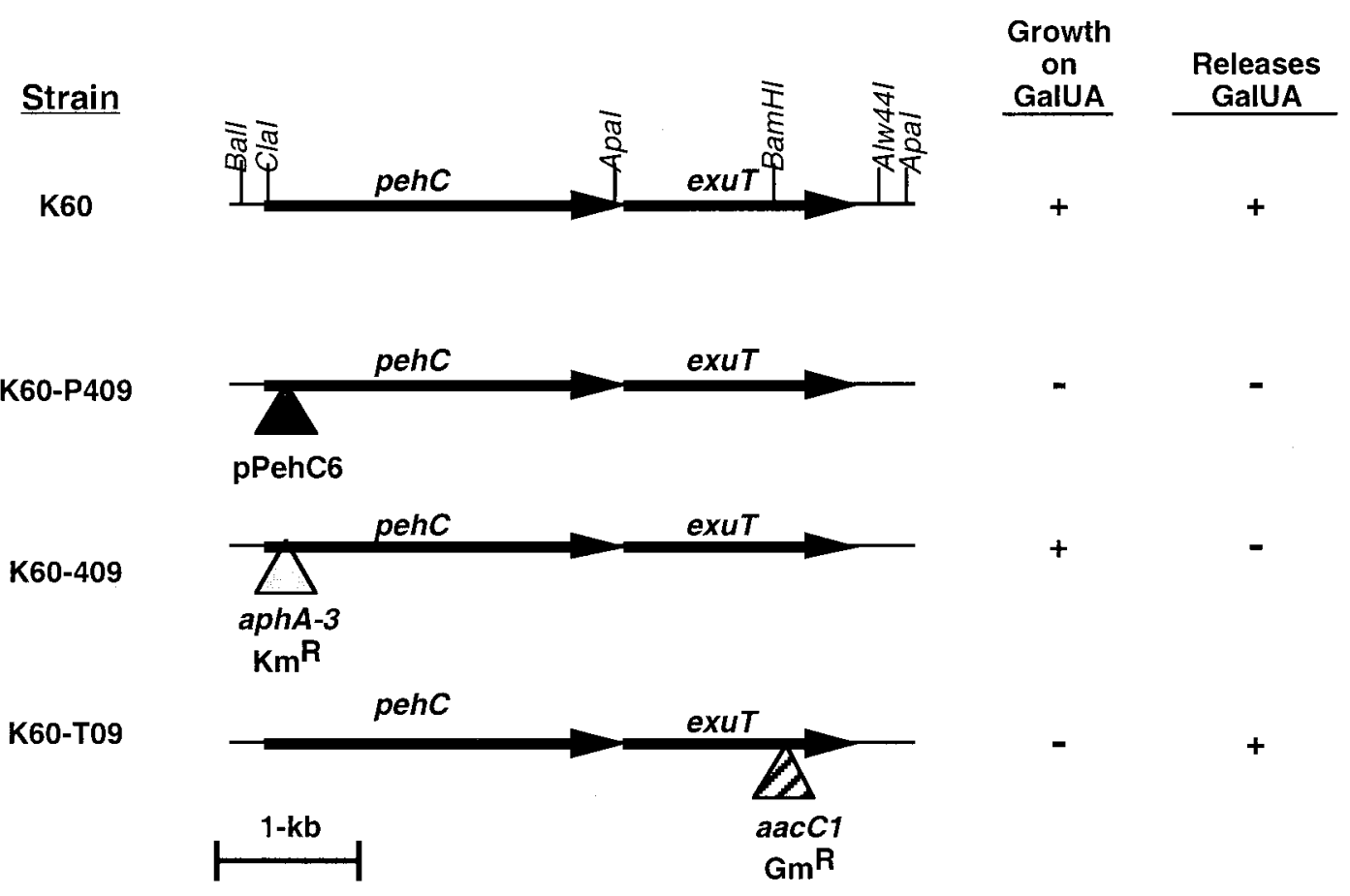

Fig. 2. Physical map and functional analysis of pehC-exuT operon. PehC activity was measured by thin layer chromatography to detect galacturonate (galUA) release from polygalacturonate. ExuT activity was measured as bacterial growth on minimal media plates amended with galUA as a sole carbon source. Restriction enzyme sites used for cloning and subcloning pehC and exuT also are indicated on this map.

A

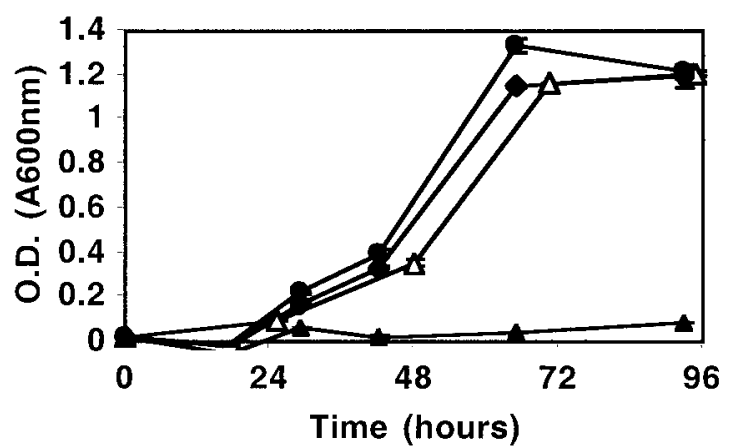

B

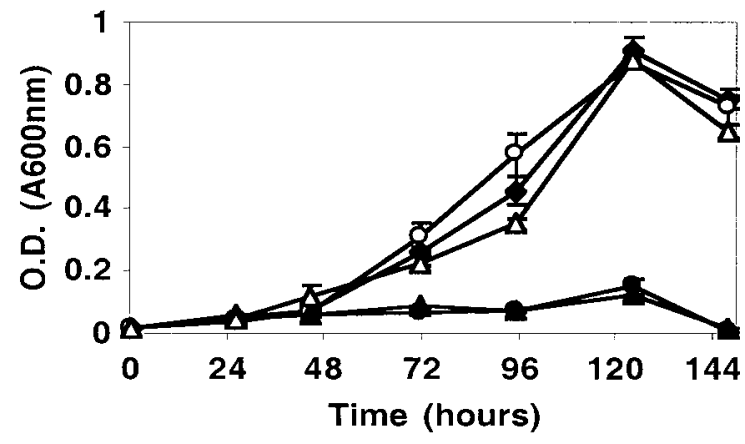

Fig. 3. Growth of pehC mutant K60-409 and exuT mutant K60-T09 on various carbon sources. A, Cultures starting at an optical density with absorbance at $600 \mathrm{~nm}\left(\mathrm{OD} \mathrm{A}_{600}\right)$ of 0.02 were grown in modified Boucher's minimal media without yeast extract (BMM) plus $0.2 \%$ (wt/vol) galacturonic acid for 96 $\mathrm{h}$ at $28^{\circ} \mathrm{C}$ with shaking. Closed diamonds, wild-type strain K60; closed triangles, exuT mutant K60-T09; open triangles, K60-T09 carrying the pehC-exuT operon in trans on pPehC11; closed circles, pehC mutant K60-409. B, Cultures starting at OD $\mathrm{A}_{600}=0.02 \mathrm{were}$ grown in BMM plus 0.2\% (wt/vol) polygalacturonic acid (potassium salt) for $176 \mathrm{~h}$ at $28^{\circ} \mathrm{C}$ with shaking. Closed diamonds, wild-type strain K60 plus empty vector pLAFR3; closed triangles, exuT mutant K60-T09 plus empty vector pLAFR3; open triangles, K60-T09 carrying the pehC-exuT operon in trans on pPehC11; open circles, pehC mutant K60-409 carrying the pehC-exuT operon in trans on pPehC11; closed circles, pehC mutant K60-409 plus empty vector pLAFR3. Bacterial growth was measured spectrophotometrically as OD $\mathrm{A}_{600}$. Results shown are the average of three replications; bars indicate standard error. 
(Fig. 3B). Interestingly, although pehC mutant K60-409 grew like the wild type in planta (data not shown), it also could not grow on PGA as a sole carbon source; however, growth was restored by adding the pehC-exuT operon in trans (Fig. 3B). This result indicated that, although this mutant could still generate di- and tri galacturonate and had intact ExuT function (as indicated by its growth on galUA), it could not take up any larger galacturonate oligomers via ExuT or any alternate sugar transporters. Thus, the only means by which $R$. solanacearum could generate a usable carbon source from PGA was with monogalacturonate-releasing PehC. These results established that exuT mutant K60-T09 could degrade PGA down to monomers but could not transport any PG degradation products, effectively separating the macerating and the nutrient-generating functions of $R$. solanacearum's PGs.

Neither galacturonic acid uptake nor PehC activity is required for wild-type virulence of $R$. solanacearum.

The virulence of pehC mutant K60-409 and exuT mutant K60-T09 was tested on tomato using two different virulence assays. Susceptible tomato seedlings, 14 to 15 days old, were inoculated by pouring a dilute bacterial suspension directly onto the soil surrounding the crown, resulting in a final bacterial soil population similar to those observed in naturally infested field during epidemics (Devi et al. 1982; Hayward 1991). This method mimics the natural infection process, when bacteria must overcome natural barriers such as soil and intact root systems to locate and invade plant roots, colonize host xylem vessels, and cause disease. Under these conditions, both K60-T09 and K60-409 had virulence that was indistinguishable from that of the wild-type parent strain by repeated measures analysis of variance $($ ANOVA $)(P$ value $=$ 0.9072) (Fig. 4A). Similarly, when these mutants were inoculated directly into wounded host plants via cut petioles, there was no difference in virulence between the exuT mutant, the pehC mutant, and the wild-type parent strain (data not shown).

Nonpectolytic mutant K60-709 is less virulent than wild-type K60, yet slightly more virulent than PehA/PehB double mutant K60-309.

Although less virulent than its wild-type parent $(P$ value $<$ 0.001), completely PG-deficient mutant K60-709 was signifi-

A

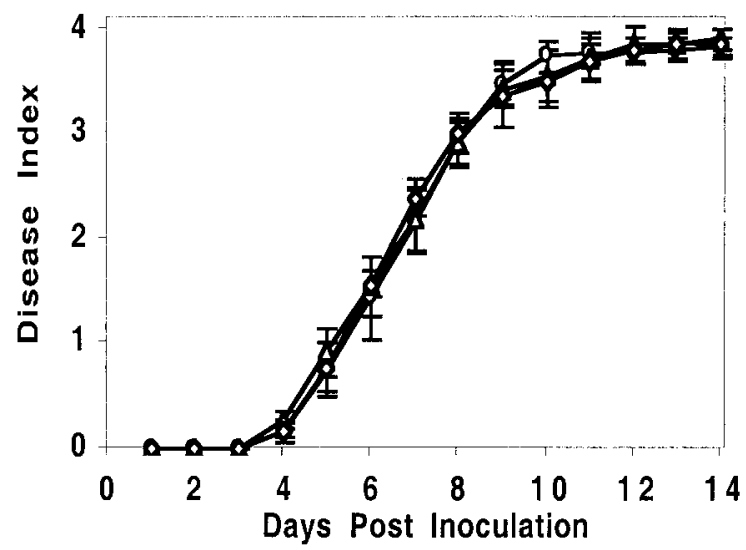

cantly more virulent on tomato than $p e h A / p e h B$ mutant K60309 ( $P$ value $<0.001)$ in our soil-soak inoculation assay (Fig. 4B). A pehR mutant, a pleiotropic regulatory mutant severely attenuated in the production of PGs as well as other virulence factors, was used as a negative control for these experiments (Allen et al. 1997).

\section{DISCUSSION}

We previously found that two of $R$. solanacearum's three extracellular PGs, endo-PG PehA and exo-poly- $\alpha-\mathrm{D}-$ galacturonosidase PehB, are necessary for full virulence of this pathogen (Huang and Allen 1997). However, we could not determine the collective role of PGs in bacterial wilt because a pehApehB double mutant still produces an extracellular exo-PG, PehC. Repeated efforts to isolate the third PG gene, pehC, using polymerase chain reaction (PCR) primers based on sequences conserved in bacterial endo-PGs and exo-poly- $\alpha$-D-galacturonosidases were unsuccessful. We eventually identified the gene by BLASTX search of the GMI1000 genome because the PehC amino acid sequence contained a few conserved motifs that suggested it might be a PG similar to those found in bacteria, plants, and fungi, although very different from previously characterized $R$. solanacearum PehA and PehB. Indeed, the PehC amino acid sequence does not strongly resemble PehB or other known exopoly- $\alpha$-D-galacturonosidases, indicating that true galUA-releasing exo-PGs are structurally distinct from their digalacturonateand oligogalacturonate-releasing cousins. Although they share a hydrolytic mechanism for degrading the polygalacturonate backbone, further CLUSTAL sequence analysis revealed that PehC also does not closely resemble known fungal exo-PG protein sequences. Fungal exo-PGs are more closely related to fungal endo-PGs than to $R$. solanacearum exo-PG PehC.

Endo-PG PehA was known to contribute substantially to bacterial wilt virulence, while exo-poly- $\alpha$-D-galacturonosidase PehB plays a smaller, though still significant, role (Huang and Allen 1997; Schell et al. 1988). Mutant strains lacking PehA and PehB are significantly reduced not only in global virulence but also in both the frequency and the speed with which they colonize tomato stems (Huang and Allen 2000). These two enzymes, especially PehA, can rapidly depolymerize the polygalacturonate backbone of plant pectins. However, we found that PehC, which only trims galUA monomers from the ends of

B

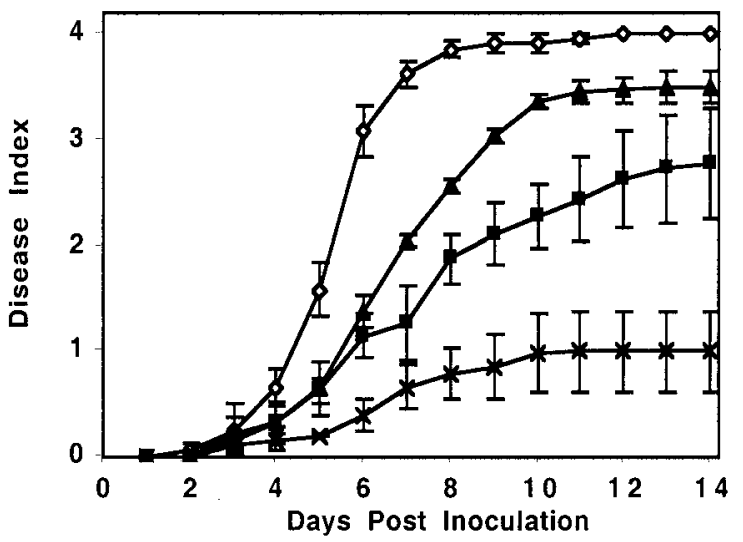

Fig. 4. Virulence of pehC mutant K60-409, polygalacturonases mutant K60-709, and exuT mutant K60-T09 on tomato. Tomato (Lycopersicon esculentum cv. Bonny Best) seedlings, 14 to 15 days old, were soil-soak inoculated with $1.5 \times 10^{7}$ to $3.1 \times 10^{7} \mathrm{CFU} / \mathrm{g}$ of soil and monitored daily for disease development. Plants were rated daily on a disease index scale ranging from 0 (healthy) to 4 (76 to 100\% leaves wilted). A, pehC Mutant K60-409 (open circles), exuT mutant K60-T09 (open triangles), and wild-type strain K60 (open diamonds). B, pehA/pehB Mutant K60-309 (closed squares), pehApehBpehC mutant K60-709 (closed triangles), pehR mutant K71(cross marks), and wild-type K60 (open diamonds). Points shown in each panel are the average of three separate experiments containing at least 16 plants/treatment/assay. Bars indicate standard error. 
pectic polymers, is dispensable for bacterial wilt virulence in our soil and petiole inoculation assays on tomato plants. Collectively, these results suggest that the primary virulence function of $R$. solanacearum's extracellular PGs is rapid maceration of plant pectic substances to facilitate pathogen invasion and spread. Nonetheless, the bacterium synthesizes and secretes relatively large amounts of PehC, suggesting that the enzyme somehow contributes to bacterial fitness.

Curiously, a mutant lacking all three PGs was more virulent than the PehA/PehB double mutant, although the onset of wilting symptoms occurred at the same time (day 4). The reason for this small yet significant difference in virulence is not clear. It is known that the oligogalacturonides released by pectolytic enzymes are powerful elicitors of plant defense responses (Esquerre-Tugaye et al. 2000; Ridley et al. 2001; Vidal et al. 1998). Specifically in tomato, oligogalacturonides can elicit production of active oxygen species, and galacturonic acid dimers and trimers can elicit production of protease inhibitors (Farmer et al. 1990; Moloshok et al. 1992). Plant cell walls are known to contain dormant plant PGs that can be released during pathogenesis (for example, by the action of Egl, the endoglucanase whose activity is present in all our PG mutant strains); these plant PGs may release some oligogalacturonide signal molecules during pathogenesis (Prade et al. 1999). Our experiments demonstrate that PehC cleaves small oligomers of galacturonate; we hypothesize that this extracellular exo-PG efficiently degrades the most potent oligogalacturonate signals, which would reduce tomato antimicrobial responses and, thus, indirectly increase bacterial virulence. We speculate that the PehA/PehB double mutant, which retains full PehC activity, still degrades some oligogalacturonides that trigger plant recognition defense gene expression. Thus, this double mutant is not only less able to colonize and spread because it lacks endoPG activity, but also still elicits host plant defense responses, resulting in reduced virulence. However, the triple mutant is completely unable to degrade oligogalacturonides and, thus, may be slightly more virulent than the PehA/B double mutant because it is less visible to its plant host. Comparison of plant defense gene induction in response to the various PG-deficient and wild-type strains could test this hypothesis and further elucidate the complex interaction between host responses and pathogen pectolytic activity.

Immediately downstream of the pehC ORF, we found a gene that resembled galUA transporter ExuT. Mutation of the exuT locus destroyed the ability of $R$. solanacearum to grow on either PGA or galUA as a sole carbon source, a phenotype consistent with galacturonate transport function. Moreover, the $R$. solanacearum exuT gene could heterologously complement an E. chrysanthemi exuT mutant, thereby confirming the biological function of the protein. Interestingly, $R$. solanacearum exuT mutant K60-T09 still produces wild-type levels of all three extracellular PGs, indicating that intracellular galUA is not required for induction of PG gene expression (see below). We found that pehC and exuT are co-transcribed. It is logical that the enzyme that releases a sugar would be co-regulated and coexpressed with its specific transporter; nevertheless, this particular arrangement is unique to $R$. solanacearum and not known in other bacterial plant pathogens. E. chrysanthemi, which produces no exo-PG activity, transcribes exuT in a monocistronic transcription unit in response to galUA (Haseloff et al. 1998).

Even though the exuT gene from $R$. solanacearum can heterologously complement an E. chrysanthemi exuT mutation, ExuT plays different biological roles in the two plant pathogens. E. chrysanthemi also can use monomers of pectic polymers as carbon and energy sources but, unlike $R$. solanacearum, E. chrysanthemi produces two types of monomers from pectin: galUA and 5-keto-4-deoxyuronate, which are actively transported into the bacterial cell by the exuT and $k d g T$ transport systems, respectively. Once inside the bacterial cell, these sugars are catabolized and converted into energy and into an intracellular inducer of genes in the pectin degradation pathway, including the extracellular pectate lyases that are required for soft-rot maceration (Hugouvieux-Cotte-Pattat et al. 1996). In contrast to our findings, an E. chrysanthemi exuT mutant is significantly reduced in virulence, as measured by its ability to macerate potato tissue (Haseloff et al. 1998). Because E. chrysanthemi has multiple transporters of pectin degradation products (TogT, TogMNAB, ExuT, and KdgT), an exuT mutant still can grow on PGA as a sole carbon source; therefore, it is unlikely that the decreased virulence of exuT mutants in this species is the result of nutritional limitation. The galUA transported by ExuT generates an inducer of the E. chrysanthemi pectin degradation regulon; therefore, the reduced virulence of exuT mutants is believed to result from globally reduced expression of diverse pectolytic enzymes.

The fact that wild-type $R$. solanacearum can grow on either PGA or galUA as a sole carbon source suggests that one important function of this bacterium's extracellular PGs might be to release an energy source for the bacteria during growth in planta. Certainly pectic substances are abundant and accessible to bacteria living in host xylem vessels, which are otherwise rather nutrient poor (Bialczyk and Lechowski 1995; Pegg 1985; Senden et al. 1992). We have observed that $R$. solanacearum multiplies faster in plant tissue than in any culture medium tested; we speculated that metabolism of pectins from xylem vessel walls might contribute to this rapid growth in planta. The fully pectolytic phenotype of exuT mutant K60-T09 allowed us to cleanly test the nutritional hypothesis of PG function, because this strain combined a wild-type ability to macerate pectins and plant tissues with a complete inability to take up or metabolize any of the resulting sugars. However, this hypothesis was convincingly rejected; because K60-T09 caused the same level of disease as its wild-type parent, its virulence apparently was unaffected by its inability to use galUA as a source of nutrition.

$R$. solanacearum mutants lacking either ExuT, PehC, or the extracellular pectinmethylesterase Pme all retain wild-type virulence on tomato plants (Tans-Kersten et al. 1998). Thus, this pathogen does not need the ability to demethylate pectic substances, degrade them down to monomers, uptake the monomers, or metabolize them in order to successfully invade, colonize, and wilt tomato host plants. Nonetheless, the presence of an intact metabolic pathway for utilization of pectic substances as carbon sources suggests strongly that these abilities somehow contribute to the fitness of $R$. solanacearum. What then are the biological roles of Pme, ExuT, PehC, and the intracellular metabolic pathway for degrading galUA in this species? Our virulence assay uses a single model host plant, tomato, and measures only behavior during the pathogenic stage of the bacterium's lifecycle. It is possible that $R$. solanacearum strain K60, which has a wide host range, does not require PehC, ExuT, or Pme while attacking tomato plants but does depend on these enzymes during pathogenesis on different host plants. It also is possible that our assay, which uses the highly susceptible heirloom tomato cultivar Bonny Best, may mask a role for these enzymes in virulence on more resistant hosts.

We speculate that ability to degrade and metabolize pectic substances may increase $R$. solanacearum's saprophytic fitness by contributing to its survival and multiplication in decaying plant material and in the soil. The pathogen survives over long periods in soil, suggesting that it can exploit the diverse resources available in this complex and competitive environment (Granada and Sequeira 1983; Nesmith and Jenkins 1983; Moffett 
and Wood 1984; vanElsas et al. 2000). The pectic substances from decaying plant debris found in soil offer a potential source of nutrition. Monitoring long-term soil survival of wild-type and exuT mutant strains in soil microcosms, both separately and in competition with each other, may shed light on what traits $R$. solanacearum needs to survive in soil environments. Alternatively, perhaps the galacturonate metabolic pathway helps $R$. solanacearum populations extract maximum resources from the decaying host in the end stages of disease. Our virulence assays measure host response; therefore, they would not detect any differences in bacterial fitness or population sizes late in disease or during exit from the dead host plant. Comparing pathogen populations in decaying plant material infected with wild-type and exuT mutant strains would test this hypothesis. Finally, extracellular exo-PG may help infecting bacteria rapidly degrade oligogalacturonate signal molecules that trigger plant recognition of pathogen attack, and thereby protect bacteria from host defense responses.

The conditions or signals that induce or repress expression of the pehC-exuT operon may yield clues about the role of the galacturonate metabolic pathway in the life history of $R$. solanacearum. In culture, the two-component regulator, PehSR, positively regulates all three PG genes. PehSR, in turn, is repressed by global virulence regulator $\mathrm{PhcA}$ in response to bacterial cell density, which has led to the theory that these genes are induced early in wilt disease development when bacterial populations are low, and then repressed during full-blown pathogenesis (Allen et al. 1997; Schell 2000). However, this regulatory model has not yet been tested in planta. Another trait regulated by PehSR, swimming motility, is regulated differently in planta than it is in culture (Tans-Kersten et al. 2001). Preliminary data suggest that PehSR is probably not the only regulator that affects pehC expression. A pehR mutant has only approximately 5\% wild-type PehA activity, but it retains approximately $50 \%$ of both PehB and PehC activity (Allen et al. 1997). Possibly, pehA is maximally expressed in the soil or early in disease development, whereas pehB and pehC may be induced separately by conditions in the plant host. Moreover, $R$. solanacearum produces little total PG activity per cell in rich medium (measured as reducing sugar ends generated), but this activity increases fivefold in minimal medium and a further fivefold in minimal medium supplemented with tobacco extracts. Intracellular fluids from $R$. solanacearum-infiltrated tobacco leaves contained approximately 40-fold more total PG activity per bacterial cell than was produced by cells growing in minimal medium, though some part of this PG activity may have been produced by the plant (Allen et al. 1991). These preliminary results suggest that the regulation of individual pectinase genes should be studied more precisely, using reporter gene constructs in planta and during growth in soil.

\section{MATERIALS AND METHODS}

\section{Bacterial strains.}

Bacterial strains and plasmids constructed and used in this study are listed in Table 1.

\section{Media and cultivation of bacterial strains.}

All $R$. solanacearum strains were grown at $28^{\circ} \mathrm{C}$ either in CPG broth (Hendrick and Sequeira 1984) or on TZC solid medium (Kelman 1954). To test carbon source utilization, bacteria were grown in BMM (Boucher et al. 1985), supplemented with either $0.2 \%$ (wt/vol) glucose, $0.2 \%$ (wt/vol) galacturonic acid, or $0.2 \%$ (wt/vol) polygalacturonate (potassium salt). For experiments analyzing PG reaction products, $R$. solanacearum strains were grown in BMM supplemented with $0.2 \%$ (wt/vol) citric acid as a sole carbon source. Escherichia coli and Er- winia chrysanthemi strains were grown in Luria-Bertani medium (Miller 1992). When necessary, antibiotics were added at the following concentrations: ampicillin at $50 \mu \mathrm{g} / \mathrm{ml}$, kanamycin at $25 \mu \mathrm{g} / \mathrm{ml}$, tetracycline at $15 \mu \mathrm{g} / \mathrm{ml}$, and gentamycin at $12.5 \mu \mathrm{g} / \mathrm{ml}$. In planta growth of both mutant and wild-type $R$. solanacearum strains was assayed in tobacco leaves as previously described (Tans-Kersten et al. 2001). Briefly, leaves from 3-month-old Nicotiana tabacum cv. Bottom Special were infiltrated with a bacterial suspension at $10^{7} \mathrm{CFU} / \mathrm{ml}$. Samples were collected at intervals over $48 \mathrm{~h}$ and bacteria were enumerated by dilution plating leaf homogenates on TZC supplemented with appropriate antibiotics.

\section{DNA manipulations.}

DNA cloning, mapping, sequencing, mutagenesis, Southern transfer, and PCR in both Escherichia coli and R. solanacearum were performed using standard procedures (Ausubel et al. 1995). Unless otherwise noted, chemicals came from SigmaAldrich (St. Louis) and molecular biology reagents came from Promega Corporation (Madison, WI, U.S.A.). E. coli and $R$. solanacearum strains were electroporated as previously described (Allen et al. 1991). Electroporatable cells of Erwinia chrysanthemi exuT mutant strain SF1602 were prepared by washing mid-log phase cells successively with 1 volume, 0.5 volume, 0.1 volume, and $1 \mathrm{ml}$ of sterile $10 \%$ glycerol (M. J. San Francisco, personal communication). DNA was mixed with competent cells and a $2.5-\mathrm{kV}$ pulse was applied in an Eppendorf electroporator (Eppendorf Scientific, Inc., Westbury, NY, U.S.A.). Transformants were selected on media amended with appropriate antibiotics.

\section{Cloning the pehC-exuT locus \\ from $R$. solanacearum K60.}

Using sequence data from the closely related $R$. solanacearum GMI1000, PCR primers were designed to amplify an internal portion of an ORF predicted to encode a PG similar to PGs from plants, fungi, and bacteria, but distinct from the two previously characterized $R$. solanacearum K60 PGs, PehA and PehB. The primer sequences were 5'-ATGGTCGAATTCATCGGCTG-3' and 5'-AGTGCTGCAGTTCATGATG-3'. The resulting 300-bp PCR fragment was randomly radiolabeled with $\alpha^{32} \mathrm{P}$-dCTP (NEN, Boston) and used to probe an $R$. solanacearum K60 genomic library (Tans-Kersten et al. 1998). Two of the cosmid clones hybridized to the 300-bp probe and one, p1-46, was used for further analysis. A variety of restriction enzymes were employed to generate a series of subclones in pBluescript $\mathrm{SK}(+)$ and in the broad host range vector pLAFR3 (Table 1).

\section{DNA sequencing and computer analysis.}

The DNA sequence of both strands of pehC and exuT were determined by primer walking. DNA primers were synthesized and sequencing was performed at the University of Wisconsin Biotechnology Center (Madison, U.S.A.). DNA and protein sequences were assembled and analyzed using the DNASTAR software package (DNASTAR, Madison, WI, U.S.A.). The resulting assembled gene sequences and their predicted protein sequence for the pehC-exuT operon were deposited into the GenBank database (accession AF417111).

\section{Construction of pehC and exuT mutants by site-directed mutagenesis.}

To create a nonpolar pehC mutant, the $a p h A-3$ gene cassette, which reinitiates translation downstream of its insertion site (Menard et al. 1993), was inserted into a blunt-ended unique ClaI site 280 bp $3^{\prime}$ of the pehC start site, yielding the $R$. solanacearum mutagenesis plasmid pPehC9. The aphA-3-pehC 
junctions of pPehC9 were sequenced to verify proper aphA-3 insertion. The pehC::aphA-3 construct was introduced into the $R$. solanacearum K60 genome by allelic replacement (Huang and Allen 1997). This construct also was introduced into pehA/pehB double mutant K60-309 using the same method. Southern blot analysis was performed to verify correct insertion of the aphA-3 cassette in the pehC locus in both $\mathrm{K} 60$ and K60-309 genetic backgrounds (data not shown). Putative pehC and triple PG mutants were screened on minimal medium agar supplemented with $0.2 \%$ (wt/vol) galacturonic acid as the sole carbon source to assay for intact function of the downstream exuT gene. To mutate the exuT locus, a 1.7-kb ApaI fragment containing exuT from pCR1 was subcloned into pBS $\triangle B a m H I$. The gentamycin resistance cassette (aacCl) from pUCGM was inserted into the unique BamHI site located 797 bp 3' from the putative exuT start site. The resulting exuT::aacC1 construct was introduced into the $R$. solanacearum K60 genome by allelic replacement, and insertion into the exuT locus was verified by Southern blot analysis (data not shown).

\section{Complementation of pehC and exuT mutants.}

A $3.8-\mathrm{kb} B a l \mathrm{I}-A l w 44 \mathrm{I}$ fragment from $\mathrm{p} 1-46$ that contained the pehC-exuT operon was cloned into pBS, generating pPehC10. This insert was transferred into the broad host range vector pLAFR3 as a 3.8-kb EcoRI-HindIII DNA fragment. The resulting plasmid, pPehC11, was introduced into $R$. solanacearum exuT mutant K60-T09 and into pehC mutant K60-409 by electroporation. Transformants were selected on TZC+tetracycline plates and screened for ExuT activity as growth on galacturonic acid as a sole carbon source or for PehC activity as release of monogalacturonate from polygalacturonic acid via TLC reaction product analysis (discussed below).

\section{Growth assays.}

Growth curves of wild-type $R$. solanacearum strains K60, K60-409, and K60-T09 on various carbon sources were determined as previously described (Tans-Kersten et al. 1998). Tetracycline was added at a concentration of $3.75 \mu \mathrm{g} / \mathrm{ml}$ for strains containing the pehC-exuT complementation vector pPehC11 or empty vector pLAFR3.

\section{PG activity assay.}

For the analysis of PG reaction products from Escherichia coli isolates harboring plasmids containing pehC, cells were harvested and fractionated into cell pellet and extracellular supernatant by centrifugation. Cell pellets were resuspended in $50 \mathrm{mM}$ morphonlinethanesulfonic acid (MES), pH 5.7, and sonicated on ice for three 15-s pulses at a microtip limit of 5, duty cycle of $50 \mathrm{mHz}$, to disrupt the cell membrane and release intracellular constituents. Total protein $(0.1 \mathrm{mg})$ from supernatant or from lysed cell pellets was added to $700 \mu \mathrm{l}$ of $0.4 \%$ (wt/vol) PGA substrate in $50 \mathrm{mM}$ MES, pH 5.7, and reaction products were visualized by TLC as previously described (Huang and Allen 1997). PG reaction products from $R$. solanacearum mutant and wild-type strains were analyzed by TLC as previously described (Huang and Allen 1997; Tans-Kersten et al. 1998).

\section{Virulence assays.}

The virulence of $R$. solanacearum mutant strains was measured using both petiole and soil-soak inoculation methods as described (Saile et al. 1997; Tans-Kersten et al. 1998). Briefly, 14- to 15-day-old tomato plants (Lycopersicon esculentum cv. Bonny Best) were soil inoculated by pouring a bacterial suspension in a range of $2.5 \times 10^{7}$ to $5.0 \times 10^{7} \mathrm{CFU} / \mathrm{ml}$ onto the soil around the crown area of each seedling. For petiole inocu- lation, 21- to 22-day-old tomato plants were inoculated by introducing a $2-\mu \mathrm{l}$ droplet containing approximately $1,000 \mathrm{CFU}$ directly onto the freshly cut petiole of the first true leaf. Disease progress was monitored daily for 14 days post inoculation, and rated on a 0 to 4 disease index scale by a rater blind to inoculation. Each assay contained at least 16 plants per treatment, and was repeated for a minimum of three trials.

\section{Statistical analysis.}

The experimental design used in the virulence assay is a fixed effects embedded in a factorial design. Results for the soil-soak virulence assay were analyzed using repeated measures ANOVA, an approach that takes into consideration the autocorrelation of disease index scored on consecutive days (Tans-Kersten et al. 2001). The SAS/STAT Software package (SAS Institute, Inc., Cary, NC, U.S.A.) was used for all computational statistical analysis.

\section{ACKNOWLEDGMENTS}

This research was supported by a grant from the National Science Foundation (IBN-0090692) and by the University of Wisconsin-Madison College of Agricultural and Life Sciences (Hatch Project WIS04447). E. T. González was supported by an NIH Biotechnology Training Grant (NIGMS 5 T32 GM08349). We thank J. Tans-Kersten and D. Brown for useful discussions, C. Robinson for construction of plasmid pCR1, M. J. San Francisco (Texas Tech University) for generously sharing the Erwinia chrysanthemi exuT mutant, and C. Boucher and S. Genin (INRACNRS, Toulouse, France) for prepublication access to the genomic sequence of $R$. solanacearum GMI1000.

\section{LITERATURE CITED}

Aldon, D., Brito, B., Boucher, C., and Genin, S. 2000. A bacterial sensor of plant cell contact controls the transcriptional induction of Ralstonia solanacearum pathogenicity genes. EMBO (Eur. Mol. Biol. Organ.) J. 19:2304-2314.

Allen, C., Gay, J., and Simon-Buela, L. 1997. A regulatory locus, pehSR, controls polygalacturonase production and other virulence functions in Ralstonia solanacearum. Mol. Plant-Microbe Interact. 10:1054-1064.

Allen, C., Huang, Y., and Sequeira, L. 1991. Cloning of genes affecting polygalacturonase production in Pseudomonas solanacearum. Mol. Plant-Microbe Interact. 4:147-154.

Ausubel, F., Brent, R., Kingston, R., Moore, D., Seidman, J., Smith, J., Struhl, K. 1995. Short Protocols in Molecular Biology. John Wiley and Sons, New York.

Bialczyk, J., and Lechowski, Z. 1995. Chemical composition of xylem sap of tomato grown on bicarbonate containing medium. J. Plant Nutr. 18:2005-2021.

Boucher, C., Barberis, P., Trigalet, A., and Demery, D. 1985. Transposon mutagenesis of Pseudomonas solanacearum: isolation of $\mathrm{Tn} 5$-induced avirulent mutants. J. Gen. Microbiol. 131:2449-2457.

Davis, K. R., Lyon, D. D., Darvill, A. G., and Albersheim, P. 1984. Hostpathogen interactions XXV. Endopolygalacturonic acid lyase from Erwinia carotovora elictis phytoalexin accumulation by releasing plant cell wall fragments. Plant Physiol. 74:52-60.

Denny, T. P., and Baek, S. R. 1991. Genetic evidence that extracellular polysaccharide is a virulence factor of Pseudomonas solanacearum. Mol. Plant-Microbe Interact. 4:198-206.

Devi, L. R., Menon, M. R., and Aiyer, R. S. 1982. Population threshold of Pseudomonas solanacearum at the onset of first symptoms of wilt in tomato. Indian J. Microbiol. 22:41-43.

Esquerre-Tugaye, M.-T., Boudart, G., and Dumas, B. 2000. Cell wall degrading enzymes, inhibitory proteins, and oligosaccharides participate in the molecular dialogue between plants and pathogens. Plant Physiol. Biochem. 38:157-163.

Farmer, E. E., Moloshok, T. D., and Ryan, C. A. 1990. In vitro phosphorylation in response to oligouronide elicitors: structural and physical relationships. Curr. Topics Plant Biochem. Physiol. 9:249-258.

Granada, G. A., and Sequeira, L. 1983. Survival of Pseudomonas solanacearum in soil, rhizosphere, and plant roots. Can. J. Microbiol. 29:433-440.

Hanahan, D. 1983. Studies on transformation of Escherichia coli with plasmids. J. Mol. Biol. 166:557-580.

Haseloff, B. J., Feeman, T. L., Valmeekam, M. W., Oner, F., Valachovic, 
M. S., and San Francisco, M. J. D. 1998. The exuT gene of Erwinia chrysanthemi EC16: nucleotide sequence, expression, localization, and relevance of the gene product. Mol. Plant-Microbe Interactions. $11: 270-276$

Hayward, A. C. 1991. Biology and epidemiology of bacterial wilt caused by Pseudomonas solanacearum. Annu. Rev. Phytopathol. 29:65-87.

Hendrick, C., and Sequeira, L. 1984. Lipopolysaccharide-defective mutants of the wilt pathogen Pseudomonas solanacearum. Appl. Environ. Microbiol. 48:94-101.

Huang, Q., and Allen, C. 1997. An exo-poly- $\alpha-D$-galacturonosidase, PehB, is required for wildtype virulence in Ralstonia solanacearum. J. Bacteriol. 179:7369-7378.

Huang, Q., and Allen, C. 2000. Polygalacturonases are required for rapid colonization and full virulence of Ralstonia solanacearum on tomato plants. Physiol. Mol. Plant Pathol. 57:77083.

Hugouvieux-Cotte-Pattat, N., Condemine, G., Nasser, W., and Reverchon, S. 1996. Regulation of pectinolysis in Erwinia chrysanthemi. Annu. Rev. Microbiol. 50:213-57.

Kelman, A. 1954. The relationship of pathogenicity of Pseudomonas solanacearum to colony appearance in a tetrazolium medium. Phytopathology 44:693-695.

Kester, H. C. M., Someren, M. A. K.-V., Muller, Y., and Visser, J. 1996. Primary structure and characterization of an exopolygalacturonase from Aspergillus tubingensis. Eur. J. Biochem. 240:738-746.

Kobayashi, T., Higaki, N., Yajima, N., Suzumatsu, A., Hagihara, H., Kawai, S., and Ito, S. 2001. Purification and properties of a galacturonic acid-releasing exopolygalacturonase from a strain of Bacillus. Biosci. Biotechnol. Biochem. 65:842-847.

Lang, C., and Dornenburg, H. 2000. Perspectives in the biological function and the technological application of polygalacturonases. Appl. Microbiol. Biotechnol. 53:366-375.

Liu, H., Kang, Y., Genin, S., Schell, M. A., and Denny, T. P. 2001. Twitching motility of Ralstonia solanacearum requires a type IV pilus system. Microbiology 147:3215-3229.

Markovic, O., and Janecek, S. 2001. Pectin degrading glycoside hydrolases of family 28: sequence-structural features, specificities, and evolution. Protein Eng. 14:615-631.

Menard, R., Sansonetti, P. J., and Parsot, C. 1993. Nonpolar mutagenesis of the ipa genes defines IpaB, IpaC, and IpaD as effectors of Shigella flexneri entry into epithelial cells. J. Bacteriol. 175:5899-5906.

Miller, J. H. 1992. A Short Course in Bacterial Genetics: A Laboratory Manual and Handbook for Escherichia coli and Related Bacteria. Cold Spring Harbor Press, Cold Spring Harbor, NY, U.S.A.

Moffett, L., and Wood, B. A. 1984. Populations of Pseudomonas solanacearum biovar 3 in naturally infested soil. Soil Biol. Biochem. 16:57-61.

Moloshok, T. D., Pearce, G., and Ryan, C. A. 1992. Oligouronide signaling of proteinase inhibitor genes in plants: structure-activity relationships of di- and tri-galacturonic acids and their derivatives. Arch. Biochem. Biophys. 294:

Nesmith, W. C., and S. F. Jenkins, J. 1983. Survival of Pseudomonas solanacearum in selected North Carolina soils. Phytopathology 73:13001304.

Pegg, G. F. 1985. Presidential address: Life in a black hole: the microenvironment of the vascular pathogen. Trans. Br. Mycol. Soc. 85:1-20.

Pietro, A. D., and Roncero, M. I. G. 1996. Purification and characterization of an exo-polygalacturonase from the tomato vascular wilt pathogen Fusarium oxysporum f. sp. lycopersici. FEMS (Fed. Eur. Microbiol. Soc.) Microbiol. Lett. 145:295-299.

Prade, R., Zhan, D., Ayoubi, P., and Mort, A. 1999. Pectins, pectinases, and plant-microbe interactions. Biotechnol. Genet. Eng. Rev. 16:361-389.

Ridley, B. L., O’Neill, M. A., and Mohnen, D. 2001. Pectins: structure, biosynthesis, and oligogalacturonide-related signaling. Phytochemistry
57:929-967.

Roberts, D. P., Denny, T. P., and Schell, M. 1988. Cloning of the $e g l$ gene of Pseudomonas solanacearum and analysis of its role in phytopathogenicity. J. Bacteriol. 170:1445-1451.

Saile, E., McGarvey, J., Schell, M., and Denny, T. 1997. Role of extracellular polysaccharide and endoglucanase in root invasion and colonization of tomato plants by Ralstonia solanacearum. Phytopathology 87:1264-1271.

Salanoubat, M., Genin, S., Artiguenave, F., Gouzy, J., Mangenot, S., Arlat M., Billault, A., Brottier, P., Camus, J., Cattolico, L., Chandler, M. Choisne, N., Claudel-Renard, C., Cunnac, S., Demange, N., Gaspin, C. Lavie, M., Moisan, A., Robert, C., Saurin, W., Schiex, T., Siguier, P., Thebault, P., Whalen, M., Wincker, P., Levy, M., Weissenbach, J., and Boucher, C. A. 2002. Genome sequence of the plant pathogen Ralstonia solanacearum. Nature 415:497-502.

Schell, M., Roberts, D. P., and Denny, T. P. 1988. Analysis of the Pseudomonas solanacearum polygalacturonase encoded by pglA and its involvement in phytopathogenicity. J. Bacteriol. 170:4501-4508.

Schell, M. A. 2000. Control of virulence and pathogenicity genes of Ralstonia solanacearum by an elaborate sensory network. Annu. Rev. Phytopathol. 38:263-292.

Schweizer, H. P. 1993. Small broad-host-range gentamycin resistance gene cassettes for site-specific insertion and deletion mutagenesis Bio/Techniques 15:831-833.

Scott-Craig, J. S., Cheng, Y.-Q., Cervone, F., Lorenzo, G. D., Pitkin, J., and Walton, J. D. 1998. Targeted mutants of Cochliobolus carbonum lacking the two major extracellular polygalacturonases. Appl. Environ. Microbiol. 64:1497-1503.

Scott-Craig, J. S., Panaccione, D. J., Cervone, F., and Walton, J. D. 1990 Endo-polygalacturonase is not required for pathogenicity of Cochliobolus carbonum on maize. Plant Cell. 2:1191-2000.

Senden, M. H. M. N., Van Der Meer, A. J. G. M., Limborgh, J., and Wolterbeek, H. T. 1992. Analysis of major tomato xylem organic acids and PITC-derivatives of amino acids by RP-HPLC and UV detection. Plant Soil 142:81-89.

Staskawicz, B., Dahlbeck, D., Keen, N., and Napoli, C. 1986. Molecular characterization of cloned avirulence genes from race 0 and race 1 of Pseudomonas syringae pv. glycinea. J. Bacteriol. 169:5789-5794.

Tans-Kersten, J., Guan, Y., and Allen, C. 1998. Ralstonia solanacearum pectinmethylesterase is required for growth on methylated pectin but not for bacterial wilt virulence. Appl. Environ. Microbiol. 64:49184923

Tans-Kersten, J., Huang, H., and Allen, C. 2001. Ralstonia solanacearum needs motility for invasive virulence on tomato. J. Bacteriol. 183:3597-3065.

vanElsas, J. D., Kastelein, P., Bekkum, P. V., Wolf, J. M. V. D., Vries, P. M. D., and Overbeek, L. S. V. 2000. Survival of Ralstonia solanacearum biovar 2, the causative agent of potato brown rot, in field and microcosm soils in temperate climates. Phytopathology 90:1358-1366.

VanGijsegem, F., Gough, C., Zischek, C., Niqueux, E., Arlat, M., Genin, S., Barberis, P., German, S., Castello, P., and Boucher, C. 1995. The hrp locus of Pseudomonas solanacearum, which encodes the production of a type III secretion system, encodes eight proteins related to components of the bacterial flagellar biogenesis complex. Mol. Microbiol. 15:1095-1114.

Vasse, J., Frey, P., and Trigalet, A. 1995. Microscopic studies of intercellular infection and protoxylem invasion of tomato roots by Pseudomonas solanacearum. Mol. Plant-Microbe Interact. 8:241-251.

Vidal, S., Ericsson, A., Montesano, M., Deneke, J., and Palva, E. T. 1998. Cell wall degrading enzymes from Erwinia carotovora cooperate in the salicylic acid-independent induction of plant defense responses. Mol. Plant-Microbe Interact. 11:23-32. 